\title{
Chromone Derivatives CM3a Potently Eradicate Staphylococcus aureus Biofilms by Inhibiting Cell Adherence
}

This article was published in the following Dove Press journal: Infection and Drug Resistance

\author{
Qing Zhan' \\ Yanlei Xu' \\ Lingling Zhan ${ }^{2}$ \\ Bingjie Wang ${ }^{3}$ \\ Yinjuan Guo ${ }^{3,4}$ \\ Xiaocui $\mathrm{Wu}^{3,4}$ \\ Wenxiu $\mathrm{Ai}^{5}$ \\ Zengqiang Song ${ }^{6}$ \\ Fangyou $\mathrm{Yu}^{3,4}$
}

'Jiangxi Provincial Key Laboratory of Preventive Medicine, School of Public Health, Nanchang University, Nanchang, 330006, People's Republic of China; ${ }^{2}$ Department of Laboratory Medicine, The First Affiliated Hospital of Wenzhou Medical University, Wenzhou, 325000, People's Republic of China; ${ }^{3}$ Department of Clinical Laboratory Medicine, Shanghai Pulmonary Hospital, Tongji University School of Medicine, Shanghai, 200082, People's Republic of China; ${ }^{4}$ Shanghai Key Laboratory of Tuberculosis, Shanghai Pulmonary Hospital, Tongji University School of Medicine, Shanghai, 200082, People's Republic of China; ${ }^{5}$ Department of Respiratory Medicine, The First Affiliated Hospital of Wenzhou Medical University, Wenzhou, 325000, People's Republic of China; ${ }^{6}$ School of

Pharmaceutical Sciences, Wenzhou Medical University, Wenzhou, 325000 , People's Republic of China
Correspondence: Fangyou Yu;

Zengqiang Song

Tel +86-021-65II5006;

+86-577-86699396

Email wzjxyfy@163.com;

songzengqiang09@163.com
Introduction: The ability of Staphylococcus aureus to form biofilms is associated with high mortality and treatment costs. Established biofilms cannot be eradicated by many conventional antibiotics due to the development of antibiotic tolerance by $S$. aureus. Here we report the synthesis and biological characterization of novel small-molecule compounds with antibiofilm activity. Chromone 5-maleimide substitution compounds (CM3a) showed favorable antibacterial activity against $S$. aureus.

Methods: CM3A with antibacterial activity was synthesized and screened. The minimum inhibitory concentration (MIC) of CM3a were determined by the broth microdilution method. Biofilm eradication assay and colony count methods were used to investigate the effect of CM3a on $S$. aureus biofilm disruption and killing. Changes in biofilm architecture when subjected to $\mathrm{CM} 3 \mathrm{a}$, were visualized using confocal laser scanning microscopy (CLSM). CCK-8 assay and survival rate of Galleria mellonella larvae were used to test the toxicity of CM3a.

Results: The minimum inhibitory concentration (MIC) of CM3a against $S$. aureus was about $26.4 \mu \mathrm{M}$. Biofilm staining and laser scanning confocal microscopy analysis showed that CM3a eradicated $S$. aureus biofilms by reducing the viability of the constituent bacterial cells. On the other hand, CM3a showed negligible toxicity against mouse alveolar epithelial cells and Galleria mellonella larvae.

Conclusion: Chromone derivatives CM3a has therapeutic potential as a safe and effective compound for the treatment of $S$. aureus infection.

Keywords: chromone derivative, maleimide, Staphylococcus aureus, biofilm, eradicate

\section{Introduction}

Staphylococcus aureus is a Gram-positive opportunistic human pathogen that causes many community-acquired, nosocomial, and biofilm-related infections worldwide. ${ }^{1}$ Implanted medical devices (eg, joint prostheses, fluid shunts, and pacemakers) and catheters (eg, intravenous, urinary, and dialysis catheters) have revolutionized modern healthcare and are widely used in clinical practice. However, they are often associated with chronic and recurrent infections caused by $S$. aureus biofilms that form on the device surface. ${ }^{2}$ Biofilms are produced upon switching the bacterial growth mode from planktonic to sessile, the process of its formation comprises 3 main stages: attachment, maturation, and detachment. ${ }^{3}$ In the detachment phase, cell clusters or a single cell separates from the biofilm, spreading to and colonizing other parts of the host. ${ }^{4,5}$ Bacterial biofilms are key players in the development of highly refractory to 
antibacterial agents because of the complex structure of the extracellular polymeric substances (EPS) matrix. ${ }^{6}$ Biofilm formation can lead to the persistence and recalcitrant of $S$. aureus and is a major clinical challenge. ${ }^{7}$ Currently employed antibiotics mainly target microbial growth mechanisms and cell division, while bacterial biofilms resist clearance by antibacterial agents and host defense molecules. Thus, bacterial biofilms increase the risks of morbidity and mortality in patients, as well as treatment costs. ${ }^{8,9}$

In several decades, although many non-growth-altering biofilm inhibitors and dispersal agents have been described, there are few biofilm-eradicating agents. ${ }^{10}$ It is impendency to develop new biofilm eradication agents and complementary antimicrobial strategies with multiple therapeutic applications to address persistent bacterial infections and reduce patient mortality and treatment costs.

Chromone is a natural compound present in the diet of humans and animals that has low toxicity to mammalian cells. ${ }^{11,12}$ Previous studies have also shown that chromone derivatives possess a broad range of biological activities ${ }^{13}$ depending on the substitution pattern of the chromone scaffold. ${ }^{14}$ Maleimide is an important structural parent nucleus of a class of bioactive molecules and functional materials from Marine natural alkaloids. ${ }^{15}$ In this study, we synthesized chromone 5-maleimide substitution compounds and assessed their therapeutic potential by characterizing their capacity to eradicate $S$. aureus biofilm.

\section{Materials and Methods}

\section{Procedure for Synthesis}

Chromone 1a ( $0.2 \mathrm{mmol}, 1$ equiv) and maleimide 2 ( 0.5 mmol, 2.5 equiv) were combined in a $12-\mathrm{mL}$ screw-capped tube with $2 \mathrm{~mL}$ of 1.2-dichloroethane $(0.1 \mathrm{M})$. [Ru(p-cymene) $\mathrm{Cl} 2] 2$ ( $0.01 \mathrm{mmol}, 0.05$ equiv), AgNTf2 ( $0.04 \mathrm{mmol}, 0.2$ equiv), and $\mathrm{AgOAc}$ ( $0.6 \mathrm{mmol}, 3$ equiv) were added to the reaction mixture, which was heated to $120^{\circ} \mathrm{C}$ in a heating mantle with stirring for $2 \mathrm{~h}$. After the reaction was completed, the reaction mixture was directly loaded onto a silica gel column and purified with a petroleum ether/EtOAc solution to obtain the 3 a product ( $\geq 83 \%$ yield; $>95 \%$ purity; Figure 1 ). The full name of CM3a is 3-(7-chloro-4-oxo-4H-chrome -5-yl)-1-ethyl-1H-pyrrole-2,5-dione.

\section{Bacterial Strains and Cells}

S. aureus strains JP21, ZSA01, ZSA02, ZSA03, ZSA04, and ZSA05 were provided by the First Affiliated Hospital of Wenzhou Medical University (Wenzhou, China). S. aureus strain SA113 was a gift from the Department of Infectious Diseases and the Key Laboratory of Endogenous Infection, Shenzhen Nanshan People's Hospital (Shenzhen, China). $S$. aureus isolates were identified using a VITEK-2 automated system (bioMérieux, Marcy l'Etoile, France) according to the manufacturer's instructions. As all S. aureus strains used in this study are routinely encountered during laboratory procedures at the hospital, the Ethics Committee of Shanghai Pulmonary Hospital of Tongji University School of Medicine waived the requirement for ethics approval.

The mouse alveolar epithelial cells MALE-12 and human bronchial epithelial cell BEAS-2B was a gift from the Department of Anesthesiology, Shanghai Pulmonary Hospital, Tongji University School of Medicine (Shanghai, China).

\section{Biofilm Formation}

Overnight cultures of $S$. aureus strains JP21, SA113, ZSA01, ZSA02, ZSA03, ZSA04, and ZSA05 were diluted 1:200 in tryptic soy broth (TSB) containing $0.5 \%$ glucose (Sigma-Aldrich, St. Louis, MO, USA) and dispensed into 96-well microfilter plates (BD Biosciences, Franklin Lakes, NJ, USA). After $24 \mathrm{~h}$ of static culture at $37^{\circ} \mathrm{C}$, the wells were washed 3 times with $50 \mathrm{mM}$ phosphatebuffered saline (PBS [pH 7.2]) to remove unattached bacterial cells. Biofilms were stabilized by fixation with methanol (99.5\%) for $15 \mathrm{~min}$ and stained with $1 \%$ crystal

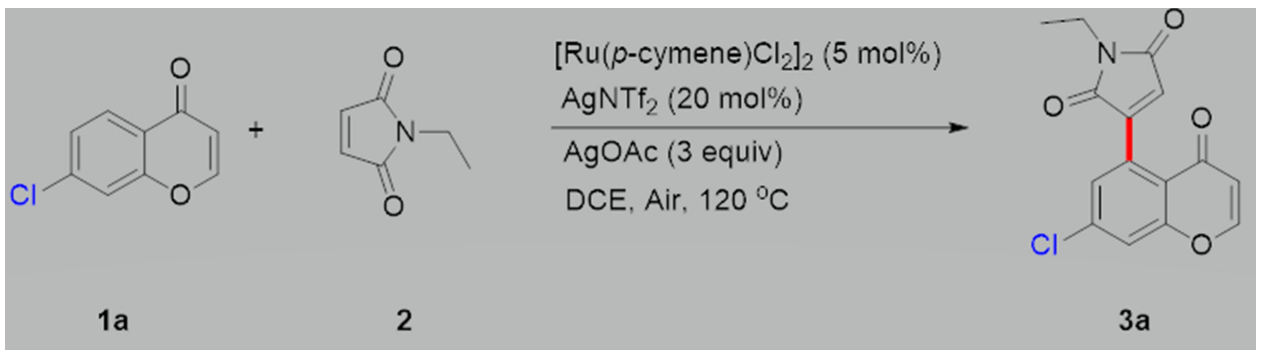

Figure I Procedure for synthesis of CM3a.Ia: chromone. 2: maleimides. 3a: CM3a. 
violet for $15 \mathrm{~min}$. The wells were gently washed 3 times with distilled water to remove floating cells. After drying, $33 \%$ glacial acetic acid was added to the well to release the biofilms into the solution, and the optical density at $600 \mathrm{~nm}$ (OD600) was measured. ${ }^{16}$

\section{Determination of Minimum Inhibitory Concentration (MIC)}

CM3a MICs for S. aureus strains JP21, SA113, ZSA01, ZSA02, ZSA03, ZSA04, and ZSA05 were determined by the broth microdilution method. Samples $(100 \mu \mathrm{L})$ were added to a 96-well microfilter plate containing CM3a $(1-512 \mu \mathrm{g} / \mathrm{mL})$ and $100 \mu \mathrm{L}$ of cation-adjusted MuellerHinton broth (CAMHB). After $24 \mathrm{~h}$ of static cultivation at $37^{\circ} \mathrm{C}$, OD600 was measured with a microplate reader (BioRad, Hercules, CA, USA). The MIC was defined as the lowest concentration that completely inhibited $S$. aureus growth.

The MICs of vancomycin, telithromycin, daptomycin for SA113 were determined by the broth microdilution method, according to the Clinical and Laboratory Standards Institute (CLSI) guidelines. ${ }^{17}$

\section{Growth Assay}

$S$. aureus strains were grown in MHB for $3 \mathrm{~h}$, then diluted 1:200 with CAMHB; $100-\mu \mathrm{L}$ aliquots were added to a 96well microfilter plate. A $100-\mu \mathrm{L}$ volume of CM3a was added to each well to obtain final concentrations of $1 / 32,1 / 16,1 / 8$, and 1/4 MIC. The fully automated Bioscreen $C$ microbial growth curve analyzer (Growth Curves USA, Piscataway, NJ, USA) was used to measure OD600 every $30 \mathrm{~min}$ for $24 \mathrm{~h}$ and a growth curve was generated from the measured values.

\section{CM3a Eradicate the Established Biofilms of $S$. aureus}

Overnight cultures of $S$. aureus were diluted 1:200 in 200 $\mu \mathrm{L}$ of TSB with $0.5 \%$ glucose (TSBG) and inoculated into a 96-well microfilter plate. After $24 \mathrm{~h}$ of static incubation at $37^{\circ} \mathrm{C}$, mature biofilms formed and the supernatant was discarded. The plate was washed with PBS to remove floating cells, and fresh TSBG containing CM3a was added to the wells; TSBG without CM3a served as the control. After static incubation for $48 \mathrm{~h}$ with daily medium replacement, the remaining biofilms were stained with crystal violet. Vancomycin, telithromycin, and daptomycin were used as control antibacterial agents for SA113 biofilms.

\section{Detecting the Adherent Cells in the Established Biofilms}

S. aureus SA113 and JP21 were inoculated into 12 polystyrene microtiter plates with TSBG and mature biofilms formed after static incubation for $24 \mathrm{~h}$. After discarding the supernatant and washing the plates 3 times, fresh TSBG containing CM3a was added to the wells; TSBG without CM3a served as an untreated control. After 48 $\mathrm{h}$ of static incubation with daily medium replacement, the adherent cells remaining in the established biofilms were collected by scratching the wall of the wells with a cell scraper after discarding the supernatant. Ultrasonic Bacteria Dispersion Counter (TB healthcare, Guangdong, China) was used to disperse bacteria of collected biofilms. The colony-forming units (CFU) of live bacteria were counted after diluting and plating the cells. ${ }^{18}$

\section{Laser Scanning Confocal Microscopy}

$S$. aureus strains were statically incubated in a $20-\mathrm{mm}$ glassbottomed cell culture dish (NEST, Wuxi, China) for 48 $\mathrm{h}$. The intermediate steps were the same as those described in Section 2.6. SYTO-9 (300 $\mu \mathrm{L}, 0.02 \%$; Thermo Fisher Scientific, Waltham, MA, USA) and propidium iodide (300 $\mu \mathrm{L}, 0.067 \%$; Thermo Fisher Scientific) were added to the cell culture dish for $30 \mathrm{~min}$ in the dark to stain the cells. The samples were imaged by laser scanning confocal microscopy (TCS SP5; Leica, Wetzlar, Germany) using a $63 \times 1.4$-numerical aperture oil immersion objective lens. Images were reconstructed into a 3-dimensional (3D) model using Imaris v7.4.2 software (Bitplane, Belfast, UK). ${ }^{19}$

\section{Assessment of CM3a Cytotoxicity}

Cell Counting Kit (CCK)-8 (Beyotime, Shanghai, China) was used according to the manufacturer's instructions to assess the viability of mouse alveolar epithelial cells MALE-12 and human bronchial epithelial cell BEAS-2B treated with CM3a. Briefly, the cells were seeded in a 96well plate at $10^{5}, 10^{4}$, and $10^{3}$ cells/well. After incubation for $48 \mathrm{~h}$ in a serum-free medium containing CM3a or PBS, the cells were incubated with $20 \mu \mathrm{L}$ CCK-8 solution for 1 $\mathrm{h}$ at $37^{\circ} \mathrm{C}$. OD 450 was measured with a Biotek Synergy 2 microplate reader (BioTek, Winooski, VT, USA). ${ }^{20}$

\section{Evaluation of CM3a Toxicity to Galleria mellonella Larvae}

As the larvae of the greater wax moth G. mellonella turn from white to black when they die, they are well-suited for 
testing drug toxicity. We injected the larvae with PBS or CM3a (16 and $32 \mu \mathrm{g} / \mathrm{mL} ; \mathrm{n}=\sim 10$ per group). The viability of the larvae was recorded after $48 \mathrm{~h}$. The experiment was performed in triplicate.

\section{Statistical Analysis}

Data were analyzed with Student's $t$-test and by 1-way factorial analysis of variance. Data analyses were performed using SPSS v19 software (IBM, Armonk, NY, USA). $P$ values $<0.05$ were regarded as statistically significant.

\section{Results}

\section{Characterization of Product CM3a}

CM3a was a white solid (50.3 mg; $>95 \%$ purity) after purification by chromatography (elution: 10\% EtOAc in petroleum ether) with a melting point of $205^{\circ} \mathrm{C}-206^{\circ} \mathrm{C}$. The compound was characterized by nuclear magnetic resonance (NMR) spectroscopy and high-resolution mass spectrometry (HRMS), yielding the following values: ${ }^{1} \mathrm{H}$ NMR $\left(400 \mathrm{MHz} \mathrm{CDCl}_{3}\right) \delta 7.81(\mathrm{~d}, J=6.0 \mathrm{~Hz}, 1 \mathrm{H}), 7.59$ (d, $J=$ $1.9 \mathrm{~Hz}, 1 \mathrm{H}), 7.22$ (d, J=1.9 Hz, 1H), 6.46 (s, 1H), 6.28 (d, $J=6.0 \mathrm{~Hz}, 1 \mathrm{H}), 3.64(\mathrm{q}, J=7.2 \mathrm{~Hz}, 2 \mathrm{H}), 1.25(\mathrm{t}, J=7.2 \mathrm{~Hz}$, $3 \mathrm{H})$ ppm. ${ }^{13} \mathrm{C}[1 \mathrm{H}] \mathrm{NMR}\left(126 \mathrm{MHz}, \mathrm{CDCl}_{3}\right) \delta 176.2,170.0$, $168.8,157.2,154.8,148.0,139.2,131.0,127.4,125.2,122.0$, 120.2, 114.0, 33.3, 13.9 ppm. HRMS (ESI-TOF) m/z: [M $+\mathrm{H}]^{+}$calcd for $\mathrm{C}_{15} \mathrm{H}_{11} \mathrm{ClNO}_{4}$ : 304.0377, found: 304.0370 .

\section{CM3a Inhibits the Growth of S. aureus}

The MIC value of CM3a against all $S$. aureus strains tested in our study was $8 \mu \mathrm{g} / \mathrm{mL}$ (about $26.4 \mu \mathrm{M}$ ). Subinhibitory concentrations of CM3a-ie, 1/4 MIC $(2 \mu \mathrm{g} / \mathrm{mL}), 1 / 8 \mathrm{MIC}$ $(1 \mu \mathrm{g} / \mathrm{mL}), 1 / 16 \mathrm{MIC}(0.5 \mu \mathrm{g} / \mathrm{mL})$, and 1/32 MIC $(0.25 \mu \mathrm{g} /$ $\mathrm{mL}$ ) - had no effect on the growth of $S$. aureus SA113 and

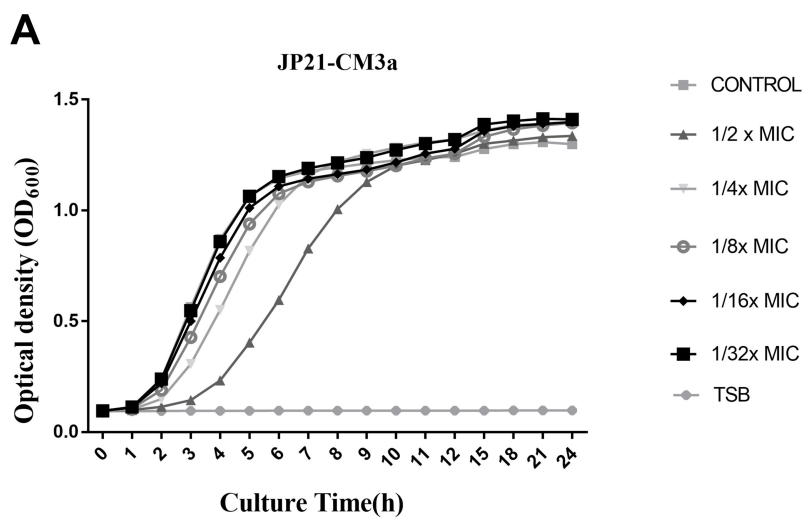

JP21. At $1 / 2 \mathrm{MIC}(4 \mu \mathrm{g} / \mathrm{mL})$, both strains grew more slowly than cells in the control group after $10 \mathrm{~h}$ (Figure 2).

\section{CM3a Eradicating the Established Biofilms of $S$. aureus}

We selected $7 \mathrm{~S}$. aureus strains that formed robust biofilms to evaluate the biofilm-inhibitory activity of CM3a. At $2 \times \mathrm{MIC}(16 \mu \mathrm{g} / \mathrm{mL})$, the OD600 of the biofilms decreased from $\sim 3$ to $\sim 1$. At CM3a concentrations of $4 \times \mathrm{MIC}(32 \mu \mathrm{g} /$ $\mathrm{mL}), 8 \times \mathrm{MIC}(64 \mu \mathrm{g} / \mathrm{mL}), 16 \times \mathrm{MIC}(128 \mu \mathrm{g} / \mathrm{mL})$, and $32 \times \mathrm{MIC}(256 \mu \mathrm{g} / \mathrm{mL})$, biofilm formation was almost completely inhibited (OD600<0.3), showing statistically significant differences relative to the control group (Figure 3A).

The MIC Values of Vancomycin, telithromycin, and daptomycin against SA113 were 1, 2, and 0.25 separately. Vancomycin, telithromycin, and daptomycin were used as control drugs to treat the biofilm of SA113. Vancomycin and daptomycin have almost no effect on the biofilm of SA113, and telithromycin has a certain effect on the concentrations of $4 \times \mathrm{MIC}$ and $8 \times \mathrm{MIC}$ (Figure $3 \mathrm{~B}$ ).

To confirm these findings, we examined the state of the biofilms after CM3a treatment with the LIVE/DEAD assay followed by laser scanning confocal microscopy. The biofilms were sparse and weak at $2 \times \mathrm{MIC}(16 \mu \mathrm{g} / \mathrm{mL})$ and almost disappeared at $4 \times$ MIC $(32 \mu \mathrm{g} / \mathrm{mL})$ (Figure 4$)$.

\section{CM3a Kills Adherent Cells in S. aureus Biofilms}

Adherent (live) cells remaining in $S$. aureus biofilms following CM3a treatment were counted. CM3a was highly effective in inhibiting the growth of adherent cells in the established biofilm. Due to the quite remarkable difference between the c. f. u. values, we used the $\log _{10}$ (c.f.u. values)

\section{B}

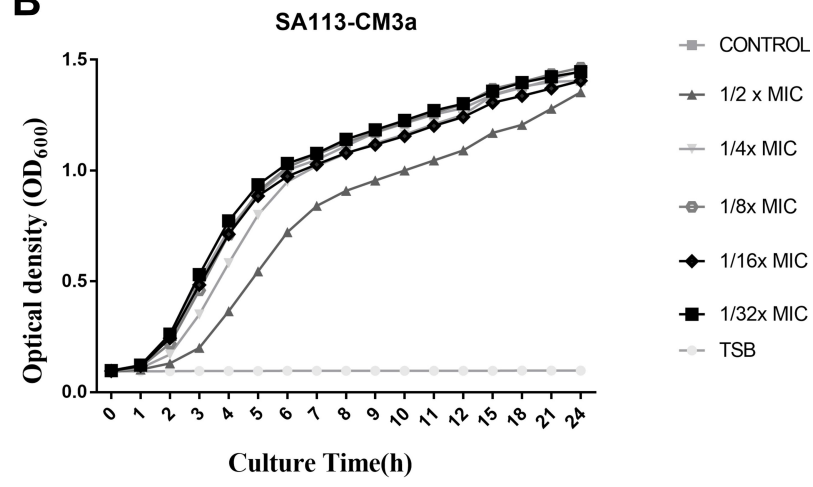

Figure 2 Growth assay for S. aureus strains treated with subinhibitory concentrations of CM3a. (A and B) Strains JP2I (A) and SAII3 (B) were cultured with 4, 2, I, 0.5, and $0.25 \mu \mathrm{g} / \mathrm{mL} \mathrm{CM3a} \mathrm{or} \mathrm{without} \mathrm{CM3a} \mathrm{for} 24 \mathrm{~h}$. 

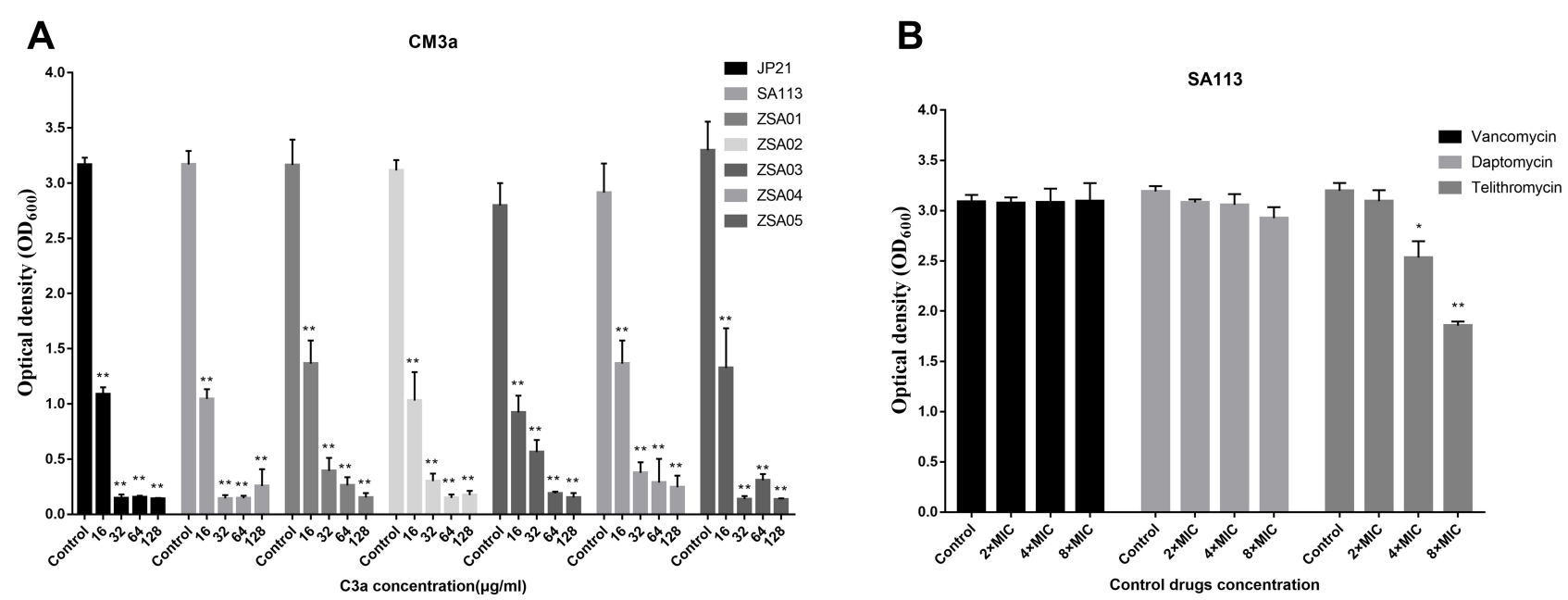

Figure 3 (A) Eradication of S. aureus (JP2I, SAII3, ZSA0I, ZSA02, ZSA03, ZSA04, and ZSA05) biofilms by CM3a (I6-128 $\mu$ g/mL). (B) Eradication of S. aureus SAII3 biofilms by vancomycin, telithromycin, and daptomycin. Each experiment was repeated 3 times, and data represent mean \pm standard deviation. $* * P<0.0 I$, $* P<0.05$.

algorithm to draw a bar graph and compare the number of remaining live bacteria. At $2 \times \mathrm{MIC}(16 \mu \mathrm{g} / \mathrm{mL})$ of CM3a, the number of live bacteria $(\mathrm{CFU} / \mathrm{mL})$ was around $10^{5}$ times lower than in the control sample; when the CM3a concentration was $\geq 4 \times$ MIC (32 $\mu \mathrm{g} / \mathrm{mL})$, the survival number of the remaining bacteria was about $10^{8}$ times lower than in the control group, living bacteria are almost gone (Figure 5).

\section{CM3a Has Low Toxicity in Mammalian Cells and Insect Larvae}

We assessed the cytotoxicity of CM3a in mouse alveolar epithelial cells MALE-12 and human bronchial epithelial cell BEAS-2B cultured with different concentrations of CM3a (16 and $32 \mu \mathrm{g} / \mathrm{mL}$ ) with the CCK-8 assay. There were no differences in survival rates between the treatment groups $(>80 \%)$ and the control group (Figure 6A and B). We also injected $G$. mellonella larvae with PBS or CM3a (16 and $32 \mu \mathrm{g} / \mathrm{mL}$ ) and evaluated their viability after 48 h. Consistent with the results of the CCK-8 assay, there were no differences in survival rates between the treatment groups and the control group (Figure 6C).

\section{Discussion}

The ability of $S$. aureus to develop robust biofilm on implanted medical devices is a major barrier to successful treatment, as biofilm bacteria are 10-1000 times more recalcitrance than planktonic bacteria to conventional antibiotics. $^{21}$ The presence of persister cells can
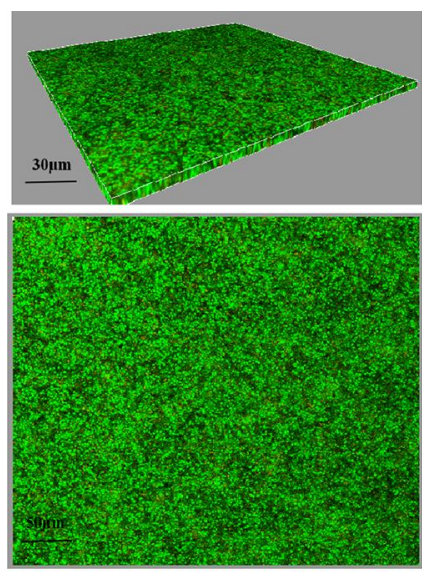

A SA113
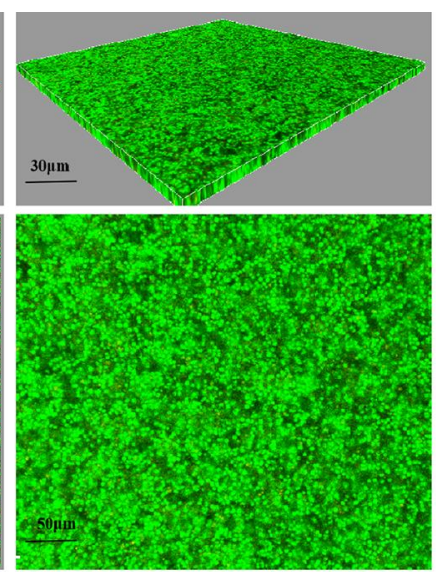

B DMSO
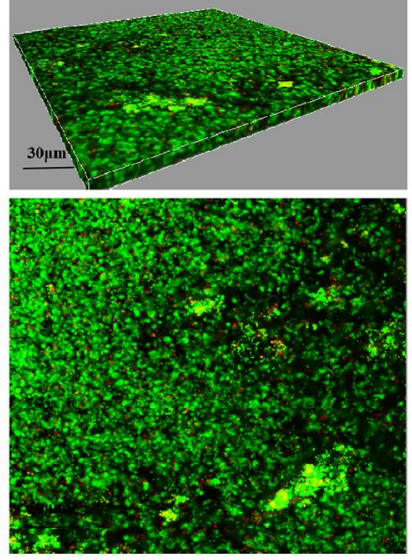

C $2 \mathrm{MIC}(16 \mu \mathrm{g} / \mathrm{ml})$

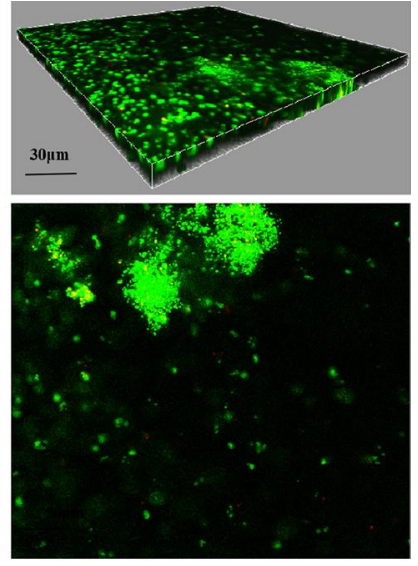

D $\quad 4 \mathrm{MIC}(32 \mu \mathrm{g} / \mathrm{ml})$

Figure 4 Biofilm formation after treatment with CM3a was observed by laser scanning confocal microscopy after the LIVE/DEAD assay. (A) Strain SAII3 without treatment. (B) SAII 3 treated with dimethylsulfoxide (DMSO). (C) SAII 3 treated with CM3a at 2 times the MIC (2×MIC). (D) SAII3 treated with CM3a at 4 times the MIC $(4 \times M I C)$. 


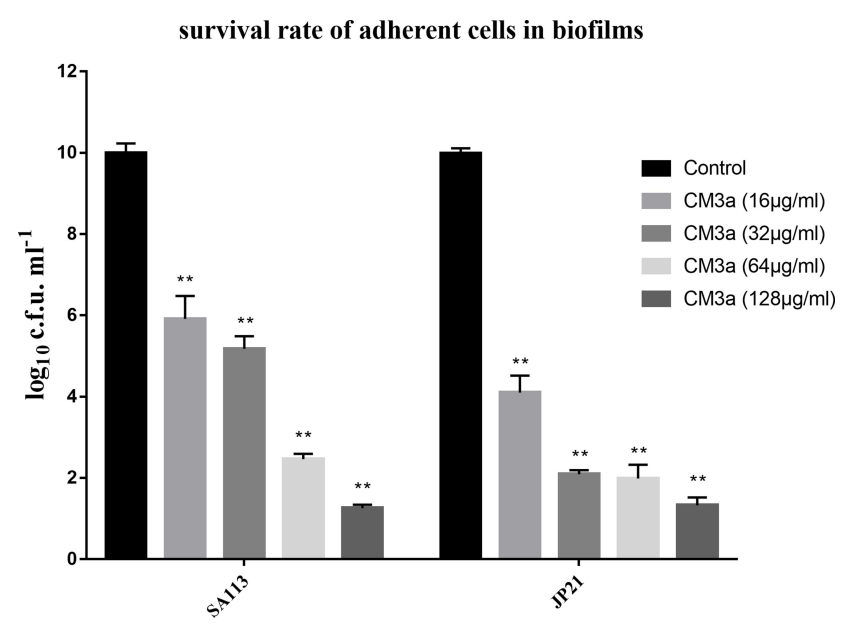

Figure 5 The survival rate of adherent cells in biofilms after treatment with CM3a $(16-128 \mu \mathrm{g} / \mathrm{mL})$. Each experiment was repeated 3 times, and data represent mean 土standard deviation. $* * \mathrm{P}<0.0 \mathrm{l}$.

disseminate and enter the circulation to colonize other organs and cause the recalcitrance and relapse of persistent bacterial infections. ${ }^{2,22}$ Many conventional antibiotics and new drugs in development stages can only inhibit the biofilm during the biofilm formation process but cannot eradicate the mature biofilm. Additionally, some antibiotics may thicken biofilms throughout treatment, making them more difficult to clear. $^{23,24}$ Therefore, new drugs are needed that can eradicate difficult-to-treat biofilms.

Chromone has a unique structure that is suited to the synthesis of new drugs. ${ }^{25}$ Chromone has been recognized as a privileged structure for the invention and development of new drugs. ${ }^{26}$ Lots of research studies prove that the Medicinal properties exhibited by chromone derivatives are antibacterial, antifungal, antioxidant, antimalarial, neuroprotective, and HIV inhibitory potential. ${ }^{13,27}$ Maleimides are the base compounds for drug conjugation to antibodies, peptides, and other targeting units through their reaction with thiol groups, and have the advantages of rapid kinetics, quantitative conversion, and high specificity. ${ }^{15}$ In our study, the chromone 5-maleimide substitution compound CM3a showed strong antibacterial activity toward $S$. aureus, with a MIC of $26.4 \mu \mathrm{M}$. In the presence of $4 \mu \mathrm{g} /$ $\mathrm{mL} \mathrm{CM} 3 \mathrm{a}$, the growth of $S$. aureus was slowed by 10 h. CM3a effectively eradicated $S$. aureus biofilms and effectively killed the constituent bacteria: the survival
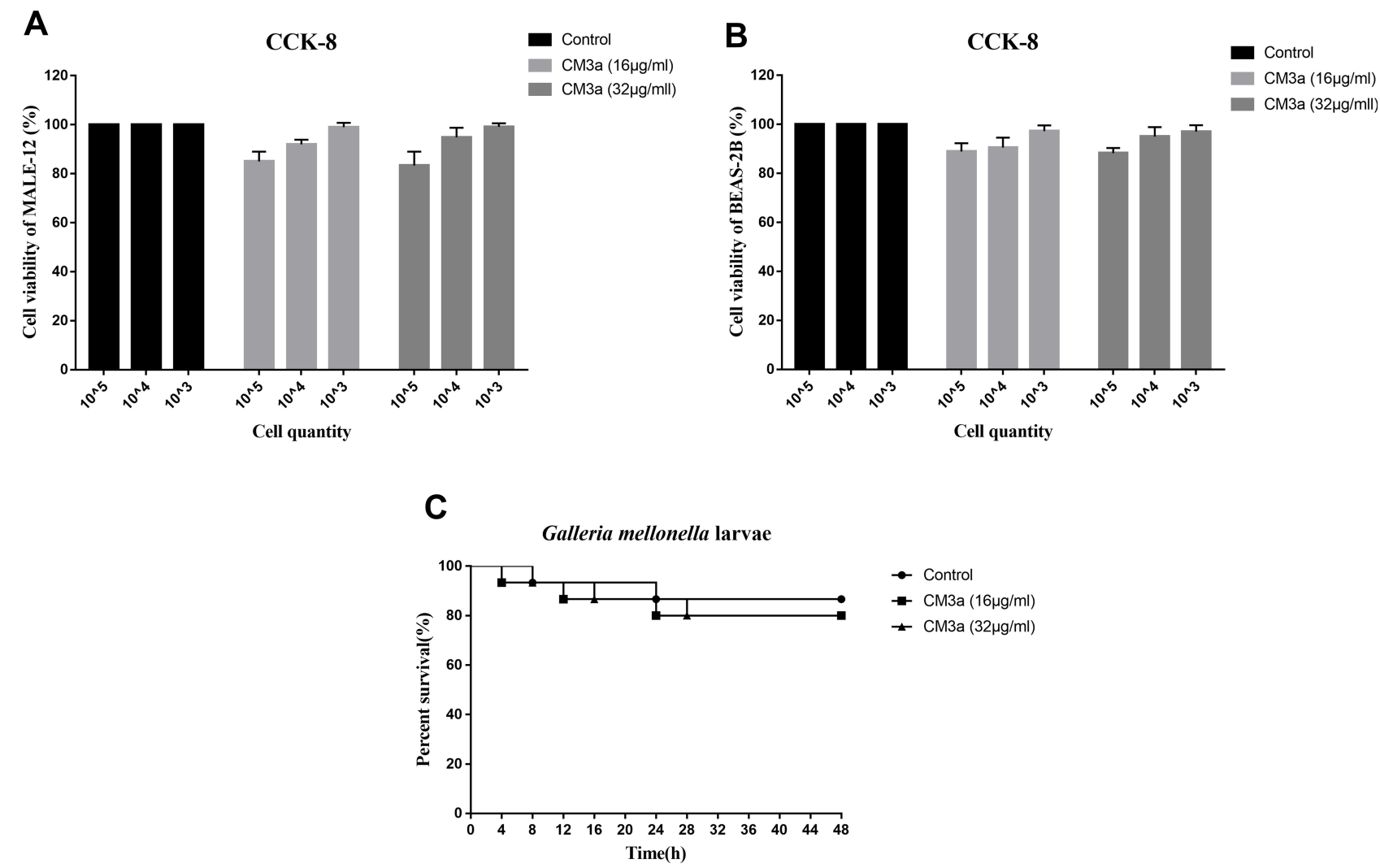

Figure 6 Toxicity of CM3a. (A and B) Viability of mouse alveolar epithelial cells MALE-12 (A) and human bronchial epithelial cell BEAS-2B (B) with or without CM3a treatment ( 16 and $32 \mu \mathrm{g} / \mathrm{mL}$ ) as determined with the CCK-8 assay. (C) Survival of $G$. mellonella larvae following injection of CM3a (I6 and $32 \mu \mathrm{g} / \mathrm{mL})$ or PBS. 
rate of the remaining live bacteria was about 105 times lower than in the control sample at CM3a concentrations $\geqq 32 \mu \mathrm{g} / \mathrm{mL}$ (about $106 \mu \mathrm{M}$ ). CM3a also promoted biofilm detachment and disintegration.

Chromone is known to have minimal toxicity to mammalian cells. We found that CM3a had low toxicity to mouse alveolar epithelial cells MALE-12 and human bronchial epithelial cell BEAS-2B, and did not cause the death of $G$. mellonella larvae. It is worth mentioning that we first synthesized the chromone derivatives without $\mathrm{Cl}$, but the solubility was poor. After modification with $\mathrm{Cl}$ or $\mathrm{Br}$, the solubility was greatly increased, and the 2 derivatives had similar antibacterial activity and capacity to eradicate $S$. aureus biofilms. Although further study is required to clarify the mechanism of action of CM3a in the destruction of biofilms, our findings show that CM3a is a promising new drug for eradicating mature $S$. aureus biofilms and thereby improving the outcomes of patients with implanted medical devices or catheters.

\section{Ethical Approval and Consent to Participate}

The Ethics Committee of the Shanghai Pulmonary Hospital of Tongji University School of Medicine approved our study. The use of all cell lines in this study was approved by the Ethics Committee of the Shanghai Pulmonary Hospital of Tongji University School of Medicine.

\section{Acknowledgments}

The authors would like to thank Yang Wu and Di Qu (Key Laboratory of Medical Molecular Virology of Ministries of Education and Health, School of Basic Medical Science and Institutes of Biomedical Sciences, Shanghai Medical College of Fudan University), Jingxin Zheng, Xiang Sun, and Zhong Chen (Department of Infectious Diseases and the Key Lab of Endogenous Infection, Shenzhen Nanshan People's Hospital and The 6th Affiliated Hospital of Shenzhen University Health Science Center), Li Zheng (the Department of Anesthesiology, Shanghai Pulmonary Hospital, Tongji University School of Medicine) for their excellent technical support and kindly suggestions.

\section{Author Contributions}

All authors contributed to data analysis, drafting or revising the article, have agreed on the journal to which the article will be submitted, gave final approval of the version to be published, and agree to be accountable for all aspects of the work.

\section{Funding}

This study was supported by grants from the Natural Science Fund of China (81871704).

\section{Disclosure}

The authors declare that there are no conflicts of interest.

\section{References}

1. Tong S, Davis JS, Eichenberger E, et al. Staphylococcus aureus infections: epidemiology, pathophysiology, clinical manifestations, and management. Clin Microbiol Rev. 2015;28(3):603-661. doi:10.1128/CMR.00134-14

2. Del Pozo J. Biofilm-related disease. Expert Rev Anti Infect Ther. 2018;16(1):51-65. doi:10.1080/14787210.2018.1417036

3. Suresh M, Biswas R, Biswas L. An update on recent developments in the prevention and treatment of Staphylococcus aureus biofilms. Int $j$ Med Microbiol. 2019;309(1):1-12. doi:10.1016/j. ijmm.2018.11.002

4. Pinto R, Soares FA, Reis S, et al. Innovative strategies toward the disassembly of the EPS matrix in bacterial biofilms. Front Microbiol. 2020;11:952. doi:10.3389/fmicb.2020.00952

5. Lister J, Horswill A. Staphylococcus aureus biofilms: recent developments in biofilm dispersal. Front Cell Infect Microbiol. 2014;4:178. doi:10.3389/fcimb.2014.00178

6. Grecka K, Xiong ZR, Chen H, et al. Effect of Ethanol Extracts of Propolis (EEPs) against staphylococcal biofilm-microscopic studies. Pathogens. 2020;9:8.

7. Del Pozo J, Patel R. The challenge of treating biofilm-associated bacterial infections. Clin Pharmacol Ther. 2007;82(2):204-209. doi:10.1038/sj.clpt.6100247

8. Conlon B. Staphylococcus aureus chronic and relapsing infections: evidence of a role for persister cells: an investigation of persister cells, their formation and their role in S. aureus disease. BioEssays: news and reviews in molecular. Cell Dev Biol. 2014;36(10):991-996.

9. Savage V, Chopra I, O'Neill A. Staphylococcus aureus biofilms promote horizontal transfer of antibiotic resistance. Antimicrob Agents Chemother. 2013;57(4):1968-1970. doi:10.1128/ AAC.02008-12

10. Hoque J, Konai MM, Gonuguntla S, et al. Membrane active small molecules show selective broad spectrum antibacterial activity with no detectable resistance and eradicate biofilms. J Med Chem. 2015;58 (14):5486-5500. doi:10.1021/acs.jmedchem.5b00443

11. Mohsin N, Irfan M, Hassan SU. et al. Current strategies in development of new chromone derivatives with diversified pharmacological activities: a review. Pharm Chem j;2020. 1-17. doi:10.1007/s11094020-02187-x

12. Legoabe L, Petzer A, Petzer J. Inhibition of monoamine oxidase by selected C6-substituted chromone derivatives. Eur J Med Chem. 2012;49:343-353. doi:10.1016/j.ejmech.2012.01.037

13. Yadav P, Parshad B, Manchanda P, et al. Chromones and their derivatives as radical scavengers: a remedy for cell impairment. Curr Top Med Chem. 2014;14(22):2552-2575. doi:10.2174/ 1568026614666141203141317

14. Matos M, Vazquez-Rodriguez S, Uriarte E, et al. Synthesis and pharmacological activities of non-flavonoid chromones: a patent review (from 2005 to 2015). Expert Opin Ther Pat. 2015;25 (11):1285-1304. doi:10.1517/13543776.2015.1078790 
15. Lahnsteiner M, Kastner A, Mayr J, et al. Improving the stability of maleimide-thiol conjugation for drug targeting. Chemistry. 2020;26 (68):15867-15870. doi:10.1002/chem.202003951

16. Sun $\mathrm{X}$, Lin $\mathrm{Z}-\mathrm{W}, \mathrm{Hu}-\mathrm{X}-\mathrm{X}$, et al. Biofilm formation in erythromycin-resistant Staphylococcus aureus and the relationship with antimicrobial susceptibility and molecular characteristics. Microb Pathog. 2018;124:47-53. doi:10.1016/j.micpath.2018.08.021

17. CLSI. Performance Standards for Antimicrobial Susceptibility Testing. 30th. 2020CLSI. CLSI supplement M100 Wayne PA: Clinical and Laboratory Standard Institute; 2018. Vol. 2018. 320.

18. Zheng J, Sun X, Lin ZW, et al. In vitro activities of daptomycin combined with fosfomycin or rifampin on planktonic and adherent linezolid-resistant isolates of Enterococcus faecalis. J Med Microbiol. 2019;68(3):493-502. doi:10.1099/jmm.0.000945

19. Bortolin M, Vecchi E, Romanò CL, et al. Antibiofilm agents against MDR bacterial strains: is bioactive glass BAG-S53P4 also effective? $J$ Antimicrob Chemother. 2016;71(1):123-127. doi:10.1093/jac/ dkv327

20. Li Y, Wang R, Xue L, et al. Astilbin protects against cerebral ischaemia/reperfusion injury by inhibiting cellular apoptosis and ROS-NLRP3 inflammasome axis activation. Int Immunopharmacol. 2020;84:106571.
21. Donlan R, Costerton J. Biofilms: survival mechanisms of clinically relevant microorganisms. Clin Microbiol Rev. 2002;15(2):167-193. doi:10.1128/CMR.15.2.167-193.2002

22. Fisher R, Gollan B, Helaine S. Persistent bacterial infections and persister cells. Nat Rev Microbiol. 2017;15(8):453-464. doi:10.1038/ nrmicro.2017.42

23. Jin Y, Guo Y, Zhan Q, et al. Subinhibitory concentrations of mupirocin stimulate staphylococcus aureus biofilm formation by upregulating cidA. Antimicrob Agents Chemother. 2020;64(3):3. doi:10.1128/ AAC.01912-19

24. Yu W, Hallinen K, Wood K. Interplay between antibiotic efficacy and drug-induced lysis underlies enhanced biofilm formation at subinhibitory drug concentrations. Antimicrob Agents Chemother. 2018;62:1.

25. Keri R, Budagumpi S, Pai RK, et al. Chromones as a privileged scaffold in drug discovery: a review. Eur $J$ Med Chem. 2014;78:340-374. doi:10.1016/j.ejmech.2014.03.047

26. Reis J, Gaspar A, Milhazes N, et al. Chromone as a privileged scaffold in drug discovery: recent advances. $J$ Med Chem. 2017;60 (19):7941-7957. doi:10.1021/acs.jmedchem.6b01720

27. Park J, Lee SU, Kim SH, et al. Chromone and chromanone derivatives as strand transfer inhibitors of HIV-1 integrase. Arch Pharm Res. 2008;31(1):1-5. doi:10.1007/s12272-008-1111-z

\section{Publish your work in this journal}

Infection and Drug Resistance is an international, peer-reviewed openaccess journal that focuses on the optimal treatment of infection (bacterial, fungal and viral) and the development and institution of preventive strategies to minimize the development and spread of resistance. The journal is specifically concerned with the epidemiology of antibiotic resistance and the mechanisms of resistance development and diffusion in both hospitals and the community. The manuscript management system is completely online and includes a very quick and fair peerreview system, which is all easy to use. Visit http://www.dovepress.com/ testimonials.php to read real quotes from published authors. 